\title{
Disulfiram-loaded porous PLGA microparticle for inhibiting the proliferation and migration of non-small-cell lung cancer
}

This article was published in the following Dove Press journal:

International Journal of Nanomedicine

24 January 2017

Number of times this article has been viewed

\author{
Chenhui Wang ${ }^{1,2, *}$ \\ Jiebing Yang ${ }^{1,3, *}$ \\ Haobo $\mathrm{Han}^{3}$ \\ Jiawen $\mathrm{Chen}^{3}$ \\ Yudi Wang ${ }^{3}$ \\ Quanshun $\mathrm{Li}^{3}$ \\ Yanbo Wang'
}

'Department of Urology, First Hospital of Jilin University, ${ }^{2}$ Innovative

Drug Research Centre, School of Pharmacy, Chongqing University,

Chongqing, ${ }^{3}$ Key Laboratory for Molecular Enzymology and

Engineering of Ministry of Education, School of Life Sciences, Jilin

University, Changchun, People's

Republic of China

*These authors contributed equally to this work

\begin{abstract}
In this study, poly(lactic-co-glycolic acid) (PLGA) was used as a carrier to construct disulfiram-loaded porous microparticle through the emulsion solvent evaporation method, using ammonium bicarbonate as a porogen. The microparticle possessed highly porous surface, suitable aerodynamic diameter for inhalation $(8.31 \pm 1.33 \mu \mathrm{m})$, favorable drug loading $(4.09 \% \pm 0.11 \%)$, and sustained release profile. The antiproliferation effect of release supernatant was detected through 3-(4,5-dimethylthiazol-2-yl)-2,5-diphenyltetrazolium bromide assay using non-smallcell lung cancer A549 as a model, with only $13.3 \%$ of cell viability observed for the release supernatant at 7 days. The antiproliferation mechanism was elucidated to be associated with the enhanced induction of cell apoptosis and cell cycle arrest at S phase through flow cytometry and Western blotting analysis. Finally, wound healing and transwell migration assay showed that they could efficiently inhibit the cell migration. These results demonstrated that disulfiramloaded porous PLGA microparticle could achieve favorable antitumor efficiency, implying the potential of treating non-small-cell lung cancer in a pulmonary administration.
\end{abstract}

Keywords: disulfiram, poly(lactic-co-glycolic acid), porous microparticle, non-small-cell lung cancer, antiproliferation, antimigration

\section{Introduction}

Non-small-cell lung cancer (NSCLC) is one of malignant diseases with 5-year survival rate of $\sim 15 \%$, owing to its diagnosis at a late stage. ${ }^{1}$ To date, its treatment is still highly dependent on chemotherapy, in which platinum-based chemotherapy is the standard first-line therapy for NSCLC patients. ${ }^{2}$ Thus, there is an urgent need to develop novel effective chemotherapeutics to overcome the limited therapeutic efficacy and strong side effects in traditional treatment, thereby increasing the overall survival of NSCLC patients. Disulfiram, a member of dithiocarbamate family, has been widely used as an antialcoholism drug in the clinics for $>60$ years. ${ }^{3}$ Recently, it has been demonstrated to possess strong antitumor ability for various tumors, including NSCLC. . $^{3-9}$ However, little progress has been achieved in in vivo antitumor studies of disulfiram, especially for oral administration, which was mainly caused by its weak stability under physiological conditions. ${ }^{10,11}$ Thus, it is of great significance to develop a carrier system for enhancing the stability of disulfiram and further facilitating the appropriate distribution to tumor tissues.

Up to now, several reports have successfully entrapped disulfiram through shellcross-linked micelle system or other formulations, which could achieve high in vitro and in vivo antitumor efficacy. ${ }^{8,11,12}$ However, these systems could not realize the 
encapsulation of disulfiram with high drug loading, and meanwhile, they were not suitable for treating lung cancers owing to their low delivery ability to lungs in an oral or intravenous administration. In contrast, inhalation systems could directly deliver the chemotherapeutics to lungs, which is beneficial for improving the distribution of therapeutic agents in tumor tissues and decreasing the systemic level of agents, thereby reducing the severity of side effects. ${ }^{13-15}$ Polymer-based porous microparticle has been considered to be an effective system for pulmonary administration owing to its unique properties, such as low density, adaptive aerodynamic diameter, and favorable lung deposition. ${ }^{16-18}$ Biodegradable polymers, such as poly(lactic-co-glycolic acid) (PLGA), have been widely used in the construction of porous microparticles for delivering many types of drugs to lungs, such as small molecules, proteins, plasmid DNA, and oligonucleotides. ${ }^{19-26}$

Herein, disulfiram-loaded porous PLGA microparticle was prepared through the emulsion solvent evaporation method, in which ammonium bicarbonate was used as a porogen. The antiproliferation and antimigration efficacy of porous PLGA microparticle against NSCLC cell line, A549 and $\mathrm{HCC} 827$, was then systematically evaluated to get a deeper insight into the mechanism.

\section{Materials and methods}

\section{Materials}

PLGA-1.5A (50:50) was purchased from Lakeshore Biomaterials (Birmingham, AL, USA), and disulfiram was purchased from Meilun Biology Technology Co., Ltd., (Dalian, People's Republic of China). Dulbecco's Modified Eagle's Medium (DMEM) and fetal bovine serum (FBS) were obtained from Gibco (Grand Island, NY, USA). Annexin V-fluoresceine isothiocyanate (FITC)/propidium iodide (PI) apoptosis detection kit and cell cycle detection kit were purchased from Bestbio (Shanghai, People's Republic of China). 3-(4,5-Dimethylthiazol-2-yl)-2,5-diphenyltetrazolium bromide (MTT) was obtained from Amersco (Solon, $\mathrm{OH}$, USA). LIVE/DEAD ${ }^{\circledR}$ Viability/Cytotoxicity kit was purchased from ThermoFisher (Grand Island, NY, USA). The antibodies against pro-caspase-3, pro-caspase-8 or caspase-8, pro-caspase- 9 or caspase- 9 and $\beta$-actin, and horseradish peroxidase (HRP)-labeled goat anti-mouse Immunoglobulin $\mathrm{G}$ were purchased from Abcam (Shanghai, People's Republic of China). Polyvinylidene fluoride (PVDF) membrane was obtained from Millipore (Billerica, MA, USA). All other reagents were of the highest grade commercially available and used as received.

\section{Preparation of porous PLGA microparticle}

Disulfiram (5 mg) and PLGA-1.5A (100 mg) were dissolved in $2 \mathrm{~mL}$ of dichloromethane, and $0.1 \mathrm{~mL}$ of ammonium bicarbonate solution $(100 \mathrm{mg} / \mathrm{mL})$ was added as a porogen. The mixture was emulsified by homogenizer at 8,000 rpm for $30 \mathrm{~s}$ in an ice bath and subsequently added to $100 \mathrm{~mL}$ of poly(vinyl alcohol) solution $(1.0 \%, \mathrm{w} / \mathrm{v})$ and homogenized at $4,000 \mathrm{rpm}$ for $2 \mathrm{~min}$. Finally, the emulsion was subjected to magnetic stirring for $3 \mathrm{~h}$ to remove dichloromethane, and the porous microparticles were collected with 500 -screen mesh, washed with distilled water three times, and lyophilized. The drug loading and encapsulation efficiency of disulfiram were measured through high-performance liquid chromatography (HPLC) on a Symmetry ${ }^{\circledR}$ C18 column $(4.6 \times 250 \mathrm{~mm})$ at room temperature, using the mobile phase of methanol and monopotassium phosphate solution $(6.8 \mathrm{~g}$ in 1,000 $\mathrm{mL}$ of distilled water, $\mathrm{pH}$ of 7.0 adjusted by $\mathrm{NaOH}$ ) at a ratio of $7: 3(\mathrm{v} / \mathrm{v})$. The elution was monitored at $250 \mathrm{~nm}$ at a flow rate of $1.0 \mathrm{~mL} / \mathrm{min}$. In the procedure, $5 \mathrm{mg}$ of porous PLGA microparticle was dissolved in $1 \mathrm{~mL}$ of acetonitrile and diluted to $2 \mathrm{~mL}$ using the mobile phase, and the supernatant was collected and applied in HPLC measurement.

\section{Characterization of porous PLGA microparticle}

Scanning electron microscopy (SEM) images of porous PLGA microparticles were taken with a JXA-840 scanning electron microscope (JEOL, Japan) with an accelerating voltage of $3 \mathrm{kV}$, in which samples were mounted on the surface of specimen stubs and coated with platinum under vacuum. The particle size and zeta potential were determined by Beckman Coulter laser scattering particle size analyzer (Brea, CA, USA) and Malvern Nano ZS90 Zetasizer (Malvern, UK), respectively. The aerodynamic diameter was determined according to the guidelines for pulmonary drug delivery in Chinese Pharmacopoeia (2010).

\section{In vitro drug release}

Briefly, $10 \mathrm{mg}$ of porous PLGA microparticles were incubated in $1 \mathrm{~mL}$ of phosphate-buffered saline (PBS; $0.01 \mathrm{M}$, $\mathrm{pH}$ 7.4) in a rotary shaker at $37^{\circ} \mathrm{C}$, and the concentration of disulfiram in the supernatants was measured during the drug release process through HPLC assay as described in the section Preparation of porous PLGA microparticle. Meanwhile, the porous microparticles were collected and analyzed through SEM to observe the changes in surface morphology. 


\section{Inhibition of cell proliferation}

The cell line (A549 and HCC827 cells) was obtained from Shanghai Institute of Cell Bank (Shanghai, People's Republic of China). The cells were seeded in 96-well plates at a density of $8.0 \times 10^{3}$ cells/well. After $24 \mathrm{~h}, 20 \mu \mathrm{L}$ of release supernatants at $1,3,5$, and 7 days were added into the wells, and the total volume in each well was maintained to $200 \mu \mathrm{L}$ using 10\% FBS-containing DMEM. After incubating for $24 \mathrm{~h}, 20 \mu \mathrm{L}$ of MTT solution $(5 \mathrm{mg} / \mathrm{mL})$ was added into each well, and the plates were incubated at $37^{\circ} \mathrm{C}$ for another $4 \mathrm{~h}$. The medium was then gently removed, and $150 \mu \mathrm{L}$ of Dimethyl sulfoxide was added into each well. The plates were agitated for $10 \mathrm{~min}$ to dissolve the formazan crystals, and the optical density at $492 \mathrm{~nm}\left(\mathrm{OD}_{492}\right)$ of each well was recorded using GF-M3000 microplate reader (Shandong, People's Republic of China). The cell viability (\%) was calculated as the ratio of $\mathrm{OD}_{492}$ values of the sample wells and control wells, respectively.

\section{Cytotoxicity assay of blank porous PLGA microparticle}

The human hepatocyte L-02 was obtained from the Cell Bank of Chinese Academy of Sciences (People's Republic of China) and cultured in DMEM supplemented with 10\% FBS at $37^{\circ} \mathrm{C}$ in a humidified atmosphere of $5 \% \mathrm{CO}_{2}(\mathrm{v} / \mathrm{v})$ in air. The cells were seeded in 96 -well plates at a density of $8.0 \times 10^{3}$ cells/well, incubated for $24 \mathrm{~h}$ and subsequently treated with different concentrations of blank porous PLGA microparticle $(0-100 \mu \mathrm{g} / \mathrm{mL})$ for $24 \mathrm{~h}$. The treatment with MTT solution and determination of cell viability were conducted as described in the section Inhibition of cell proliferation.

\section{Live/dead staining}

The A549 cells were seeded in 6-well plates at a density of $2.5 \times 10^{5}$ cells $/$ well and incubated at $37^{\circ} \mathrm{C}$ for $24 \mathrm{~h}$. Afterwards, $300 \mu \mathrm{L}$ of release supernatants at 1, 3, 5, and 7 days was added into the wells, and the total volume in each well was kept to be $2.0 \mathrm{~mL}$ using $10 \%$ FBS-containing DMEM. After incubating for $24 \mathrm{~h}$, the medium was gently removed, and the cells were harvested and washed with PBS twice. Finally, the cells were stained with the live/dead assay reagents (calcein AM and ethidium homodimer) for $20 \mathrm{~min}$, washed with $1 \mathrm{~mL}$ PBS and observed under Olympus IX71 fluorescence microscopy (Tokyo, Japan).

\section{Cell apoptosis analysis}

The A549 cells were seeded in 6-well plates at a density of $2.5 \times 10^{5}$ cells $/$ well and incubated at $37^{\circ} \mathrm{C}$ for $24 \mathrm{~h}$.
The medium was then removed, and the cells were incubated with $2 \mathrm{~mL}$ medium containing $300 \mu \mathrm{L}$ of release supernatants at 1, 3, 5, and 7 days. After $24 \mathrm{~h}$, the cells were harvested, washed with PBS twice, and suspended in binding buffer provided in the Annexin V-FITC/PI apoptosis detection kit. According to the manufacture's protocols, the cells were mixed with Annexin V-FITC and PI and incubated in the dark at room temperature for $10 \mathrm{~min}$, and the cell apoptosis was analyzed by FACSCalibur (BD Biosciences, Mountain View, CA, USA).

\section{Cell cycle arrest analysis}

The A549 cells were seeded in 6-well plates at a density of $2.5 \times 10^{5}$ cells/well and incubated at $37^{\circ} \mathrm{C}$ for $24 \mathrm{~h}$. Afterwards, the release supernatants at $1,3,5$, and 7 days $(300 \mu \mathrm{L})$ were added into the wells, and the plates were incubated at $37^{\circ} \mathrm{C}$ for another $24 \mathrm{~h}$. The cells were harvested, washed with PBS twice, and treated with $0.5 \mathrm{~mL}$ solution containing $10 \mu \mathrm{L}$ RNase A $(25 \mu \mathrm{g} / \mathrm{mL})$ and $10 \mu \mathrm{L}$ PI $(50 \mu \mathrm{g} / \mathrm{mL})$ in the cell cycle detection kit at $37^{\circ} \mathrm{C}$ for $30 \mathrm{~min}$ in the dark. The cell cycle arrest was measured by FACSCalibur (BD Biosciences).

\section{Western blotting analysis}

Briefly, the A549 cells were collected, washed with PBS twice, and lysed in the buffer solution (50 mM Tris buffer at $\mathrm{pH} 8.0,150 \mathrm{mM} \mathrm{NaCl}, 0.1 \%$ sodium dodecyl sulfate, $1.0 \%$ NP40, $0.5 \%$ sodium deoxycholate, and $1 \mathrm{mM}$ phenylmethanesulfonyl fluoride) on ice for $2 \mathrm{~h}$. The supernatants were collected through the centrifugation at $12,000 \mathrm{rpm}$ for $10 \mathrm{~min}$. An equal amount of protein was applied in sodium dodecyl sulfate-polyacrylamide gel electrophoresis and transferred to PVDF membrane by electroblotting. The membrane was blocked with PBS containing 5\% nonfat milk and $0.1 \%$ Tween-20 (PBST) at room temperature for $1 \mathrm{~h}$. After incubating with corresponding antibodies at $4{ }^{\circ} \mathrm{C}$ overnight, the membrane was washed with PBST twice and incubated with HRP-labeled secondary antibody at room temperature for $1 \mathrm{~h}$. The proteins were detected by ChemiScope 3600 chemiluminescence imaging system (Clinx Science Instrument, Shanghai, People's Republic of China).

\section{Wound healing assay}

The A549 cells were seeded into 6-well plates at a density of $3.0 \times 10^{5}$ cells/well in $2 \mathrm{~mL} 10 \%$ FBS-containing DMEM and cultured to $90 \%$ confluence. The release supernatants at $1,3,5$, and 7 days $(300 \mu \mathrm{L})$ were added into the wells, and a mechanical scratch wound was produced using 
a sterile pipette tip. The digitized images of wound area were recorded at $0,6,12$, and $24 \mathrm{~h}$ using IX71 fluorescence microscopy (Olympus).

\section{Transwell migration assay}

The cell culture and further treatment with release supernatants were performed as described in the section Wound healing assay, and the cell migration assay was conducted using Transwell chamber (Costar, Corning, NY, USA). The serum-free DMEM $(200 \mu \mathrm{L})$ containing $2.0 \times 10^{4}$ cells and $1 \%$ bovine serum albumin were added into the upper chamber of transwell with $8 \mu \mathrm{m}$ pores, whereas $600 \mu \mathrm{L}$ of $10 \%$ FBScontaining DMEM was added in the lower chamber. After the cell migration at $37^{\circ} \mathrm{C}$ for $24 \mathrm{~h}$, nonmigrating cells on the top of membrane were carefully removed by mechanical wiping. The cells that have migrated to the lower surface of membrane were fixed with $75 \%$ ethanol at $4{ }^{\circ} \mathrm{C}$ for $20 \mathrm{~min}$ and stained with $0.2 \%$ crystal violet for $15 \mathrm{~min}$. After washing with PBS three times, the cells were detected in a random objective field (200× magnification) using an Olympus IX71 fluorescence microscopy.

\section{Results and discussion}

Disulfiram-loaded porous PLGA microparticle was prepared through the emulsion solvent evaporation method, using ammonium bicarbonate as a porogen. The porous microparticles exhibited uniform sphere morphology with considerable surface porosity through SEM observation (Figure 1A and B), owing to the addition of ammonium bicarbonate, which could be decomposed into ammonia and carbon dioxide. The particle diameter of microparticles was measured to be $47.83 \pm 13.21 \mu \mathrm{m}$ using laser scattering particles size analyzer, which was consistent with SEM images. Nevertheless, the aerodynamic diameter of microparticle was determined to be $8.31 \pm 1.33 \mu \mathrm{m}(<10 \mu \mathrm{m})$, which meant that it could achieve the goal of alveolar deposition if well aerosolized and properly inhaled..$^{16,19,24}$ In addition, the porous microparticles exhibited a negative zeta potential of $-14.9 \pm 4.7 \mathrm{mV}$. Through HPLC analysis, drug loading and encapsulation efficiency of disulfiram were determined to be $4.09 \% \pm 0.11 \%$ and $81.84 \% \pm 2.35 \%$, respectively. To date, disulfiram was usually entrapped in the carrier system with relatively lower drug loading $(<5.0 \%)$, and the highest value was achieved by Song et al $(7.80 \%) .{ }^{11}$ Thus, porous PLGA microparticle in the present research could realize the entrapment of disulfiram in a relatively ideal loading. Subsequently, the drug release profile of porous PLGA microparticles was studied, and a sustained release behavior of disulfiram could be clearly observed owing to the degradation of PLGA in PBS (Figure 2). After 7 days, $~ 90 \%$ cumulative release of disulfiram could be detected. Meanwhile, the corrosion of microparticles' surface and the further collapsed backbone could be clearly observed during the drug release process (Figure 1C-F). The release supernatants at different days were then used for investigating the antiproliferation and antimigration effects on A549 cells.

Using human hepatocyte L-02 as a model, no obvious cytotoxicity was observed for blank porous PLGA microparticle with cell viability of $>90 \%$ in the selected concentration $(0-100 \mu \mathrm{g} / \mathrm{mL}$; Figure $\mathrm{S} 1)$. The antiproliferation effect on A549 and HCC827 cells induced by the release supernatants from porous PLGA microparticle was evaluated through MTT assay. As shown in Figure 3A, the release supernatants at $1,3,5$, and 7 days could obviously inhibit the proliferation of A549 cells. With the increasing concentration of disulfiram in the release supernatant, the cell viability exhibited a decreasing tendency with only $13.3 \%$ of cell viability achieved using the release supernatant at 7 days. Similarly, the release supernatants could also inhibit the cell proliferation of HCC 827 cells (Figure 3B). However, the sensitivity of $\mathrm{HCC} 827$ cell was not so sensitive to the release supernatants as A549 cells. Meanwhile, we detected the cell death induced by release supernatants through live/ dead assay (Figure 4), in which live cells are dyed green by the intracellular enzymatic hydrolysis of calcein AM and dead cells are stained red by the intercalation of ethidium homodimer to DNA. ${ }^{27}$ Clearly, compared to the control group, more cells were dyed red in the treatment groups of release supernatants, especially for those at 5 and 7 days, indicating the strong tumor cell-killing ability of supernatants from porous PLGA microparticle. In addition, cell numbers in the treatment groups showed a decreasing tendency, which could also be attributed to the cell death from the antiproliferative effect of released disulfiram.

To further elucidate the mechanism of antiproliferation effect, the cell apoptosis and cell cycle arrest induced by the release supernatants from disulfiram-loaded porous PLGA microparticles were systematically evaluated by flow cytometry. As shown in Figure 5, the ratios of early apoptotic cells in different treatment groups were measured using Annexin V/PI staining followed by flow cytometric analysis. In the control group, large viable cell population could be observed with few cells in the early apoptotic stage $(0.45 \%)$. Nevertheless, cell populations exhibited increasing number of cells at the stage of early apoptosis when the cells were treated with the release supernatants 



Figure I SEM images of disulfiram-loaded porous poly(lactic-co-glycolic acid) microparticle.

Notes: The porous surface (A and $\mathbf{B}$ ) and the surface changes during drug release at I (C), 3 (D), 5 (E) and 7 (F) days.

Abbreviation: SEM, scanning electron microscopy.

from disulfiram-loaded porous PLGA microparticle. For instance, the apoptotic ratio could achieve to $49.18 \%$ using the release supernatant at 7 days, implying the high ability of cell apoptosis induction from the released disulfiram. As the apoptosis and its related signaling pathway play an important role in the progression of cancer, ${ }^{28}$ the induction of cell apoptosis of tumor cells will be significant for the prevention and intervention of tumors, including NSCLC. Herein, the expression levels of proteins associated with cell apoptosis were assayed through Western blotting (Figures 6 and S2). The expression level of pro-caspase- 3 could be observed to be decreased in A549 cells treated with the release supernatants, indicating the activation of pro-caspase- 3 to produce active executive caspase-3 (an effector caspase) during the disulfiram-inducing cell apoptosis. Meanwhile, the release supernatants at 5 and 7 days could decrease the expression level of pro-caspase-3 at the highest level. Notably, the expression levels of pro-caspase- 8 and pro-caspase- 9 also 




Figure $\mathbf{2}$ The cumulative release of disulfiram in porous poly(lactic-co-glycolic acid) microparticle.

Note: The data were expressed as mean value \pm standard deviation, $n=3$.

decreased to produce active caspase- 8 and caspase- 9 after the treatment with release supernatants but mainly in the groups with high concentration of disulfiram. These results indicated that disulfiram in the release supernatants could induce the early apoptosis through the activation of both mitochondriamediated pathway and death receptor-mediated pathway in A549 cells. Furthermore, the cell cycle arrest of A549 cells induced by the release supernatants was monitored by PI staining and flow cytometry (Figure 7). Almost no cell cycle arrest was observed for the groups using the release supernatants at 1 and 3 days due to the lower concentration of disulfiram, whereas the release supernatants at 5 and 7 days could obviously realize the cell cycle arrest at $\mathrm{S}$ phase. For instance, $50.81 \%$ of $\mathrm{S}$ phase could be achieved using the release supernatant at 7 days. This phenomenon was consistent with the previous reports on the cell cycle arrest using disulfiram-loaded PLGA nanoparticle, ${ }^{29,30}$ which showed that disulfiram increased the number of cells accumulated in G0/G1 phase in short time and improved the number of cells accumulated in $\mathrm{S}$ phase after long-time incubation. As there are three checkpoints in S phase (the replication checkpoint, the $\mathrm{S} / \mathrm{M}$ checkpoint, and the replication-independent intraS-phase checkpoint), the disulfiram-mediated cell cycle arrest was probably caused by reactive oxygen species production and the further breakage of DNA strand. ${ }^{29,31}$

Finally, the inhibition of cell migration by the release supernatants from disulfiram-loaded porous PLGA microparticle was evaluated by wound healing and transwell migration assay. As shown in Figure 8, the control group could rapidly repair the wound through cell migration, while the wound repair was obviously blocked after treating with the release supernatants, especially at higher concentration of disulfiram (5 and 7 days). These results indicated that disulfiram-loaded porous PLGA microparticles could achieve the goal of inhibiting the cell migration of NSCLC. Transwell migration assay was used to further detect the antimigration effect, in which crystal violet staining was used to capture the representative images of migrated cells at the bottom of membrane (Figure 9). Compared to the control group, the number of migrated cells was much lower in the cells treated with the release supernatants, and the inhibitory effect showed a high dependence on the concentration of released disulfiram. Thus, disulfiram-loaded porous PLGA microparticles could successfully inhibit the cell migration and exhibited great potential in solving the metastasis-related recurrence of lung cancers.


Figure 3 The antiproliferation assay of A549 (A) and HCC827 (B) cells treated with the release supernatants at I, 3, 5, and 7 days from disulfiram-loaded porous poly(lacticco-glycolic acid) microparticles for $24 \mathrm{~h}$. The data were expressed as mean value $\pm \mathrm{SD}, \mathrm{n}=3$. Abbreviation: SD, standard deviation. 

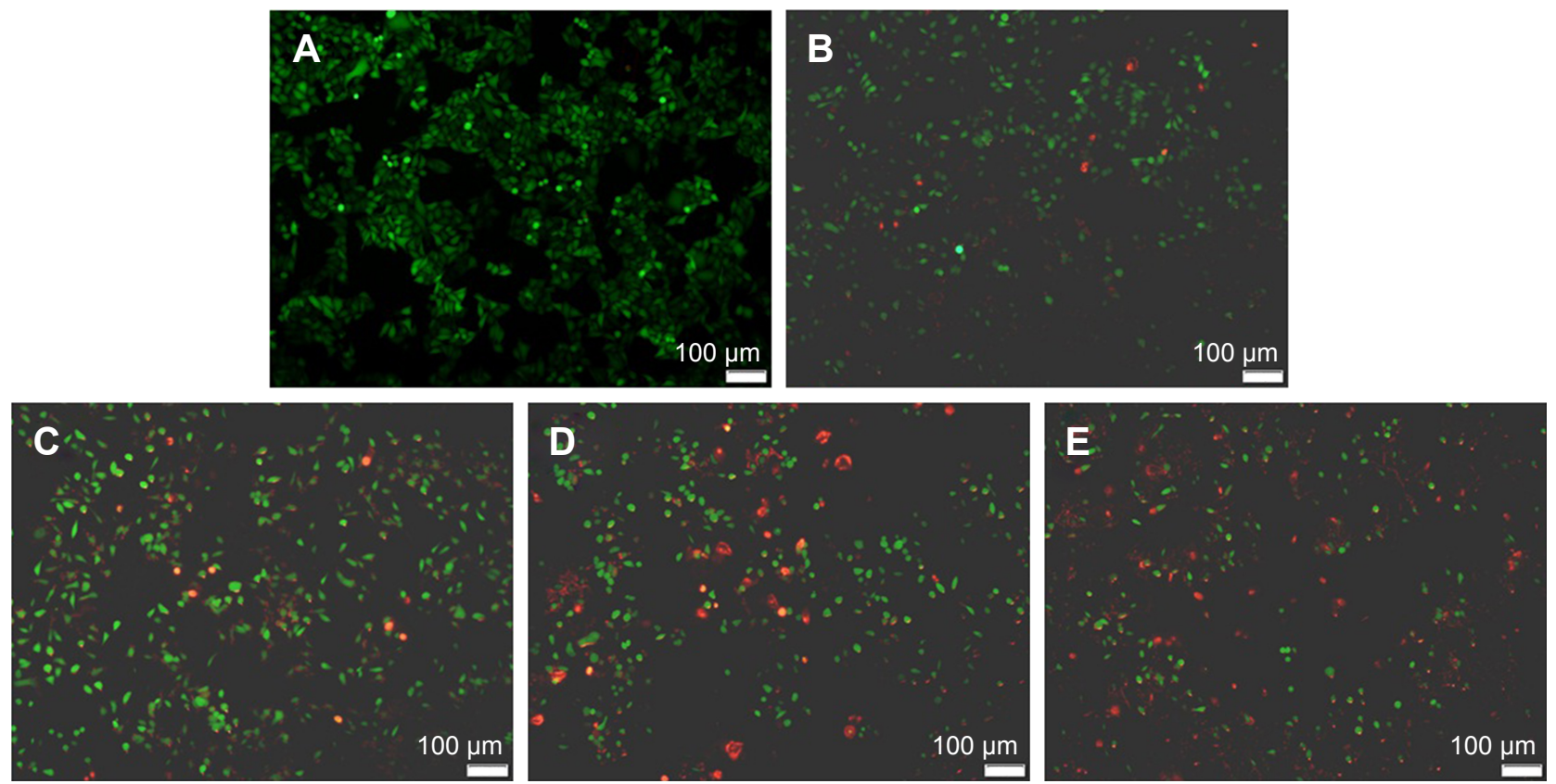

Figure 4 Live/dead assay of A549 cells after the $24 \mathrm{~h}$ treatment with the release supernatants from disulfiram-loaded porous poly(lactic-co-glycolic acid) microparticles: (A) control; (B-E) treatment with the release supernatants at I, 3, 5, and 7 days, respectively. Living and dead cells exhibited green and red fluorescence, respectively.

\section{Conclusion}

In summary, disulfiram-loaded porous PLGA microparticle was successfully prepared and characterized with highly porous surface, favorable aerodynamic diameter, excellent drug loading, and release profile. Meanwhile, it could achieve efficient antiproliferation and antimigration effects on NSCLC. Thus, it can be potentially used as a promising delivery system for lung cancer treatment through pulmonary administration, even for solving the metastasis-related recurrence of tumor.
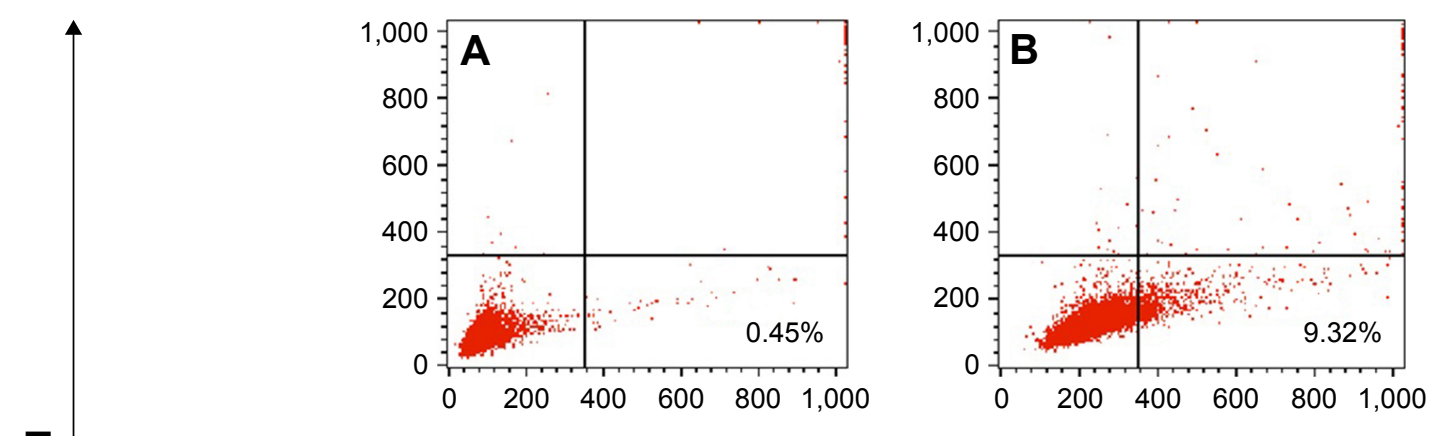

$\overline{\mathbf{\alpha}}$


Annexin V

Figure 5 Flow cytometric analysis for the cell apoptosis of A549 cells induced by the release supernatants from disulfiram-loaded porous poly(lactic-co-glycolic acid) microparticles after $24 \mathrm{~h}$ treatment: (A) control; (B-E) treatment with the release supernatants at I, 3, 5, and 7 days, respectively. Abbreviation: PI, propidium iodide. 


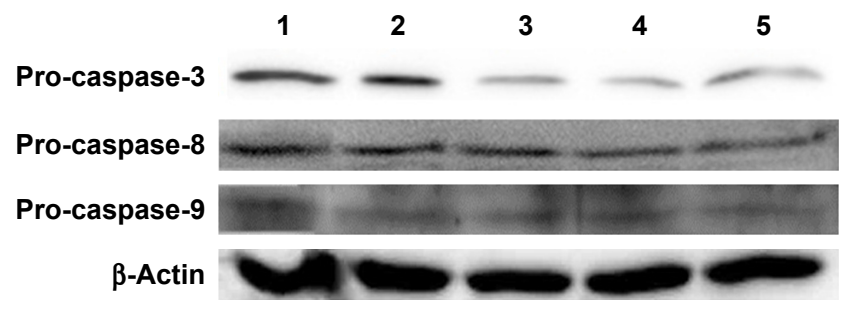

Figure 6 Western blotting analysis for the expression levels of pro-caspase-3, pro-caspase- 8 , and pro-caspase- 9 in A549 cells treated with the release supernatants from disulfiram-loaded porous poly(lactic-co-glycolic acid) microparticles for $24 \mathrm{~h}$ : lane I: control; lanes $2-5$ : treatment with the release supernatants at I, 3, 5, and 7 days, respectively.


d

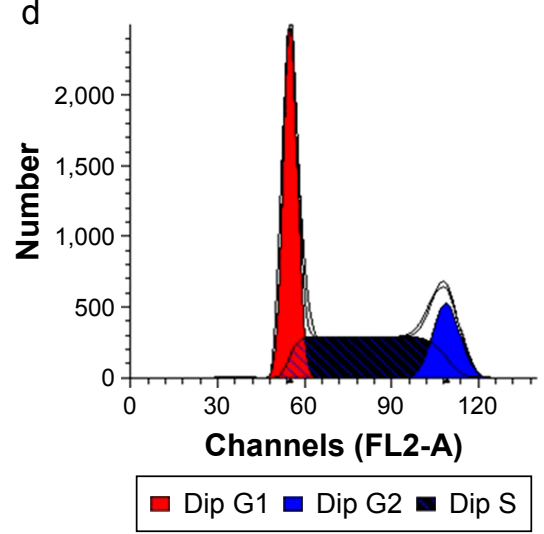

b


B

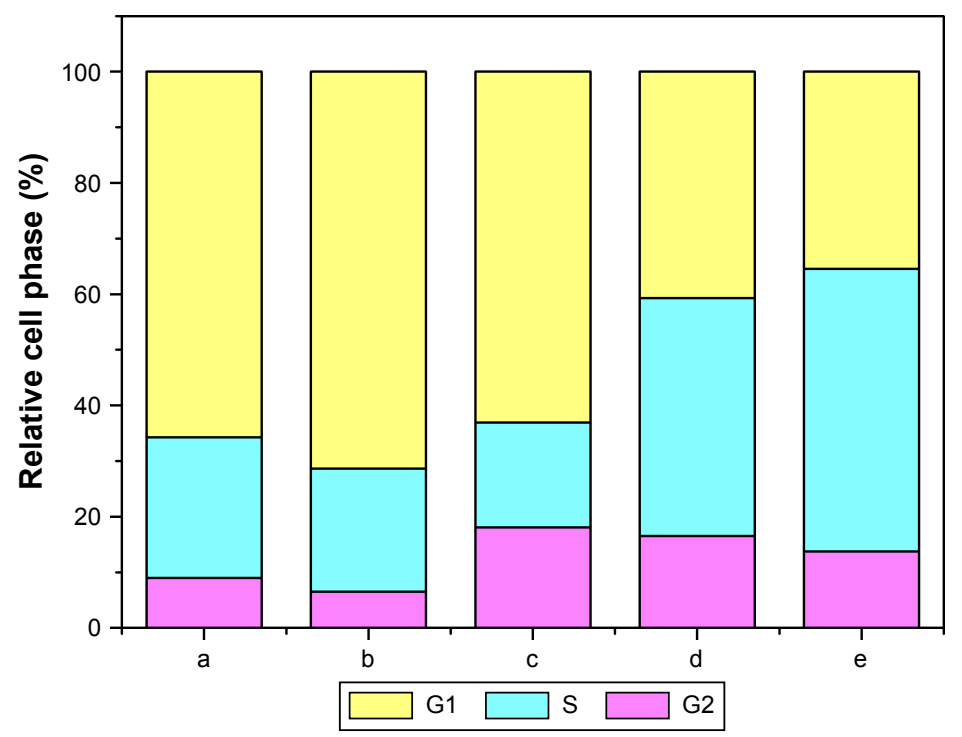

Figure 7 Cell cycle arrest (A) and relative distribution of cell population (B) in A549 cells treated with the release supernatants from disulfiram-loaded porous poly(lacticco-glycolic acid) microparticles for $24 \mathrm{~h}$ : (a) control; (b-e) treatment with the release supernatants at I, 3, 5, and 7 days, respectively. 

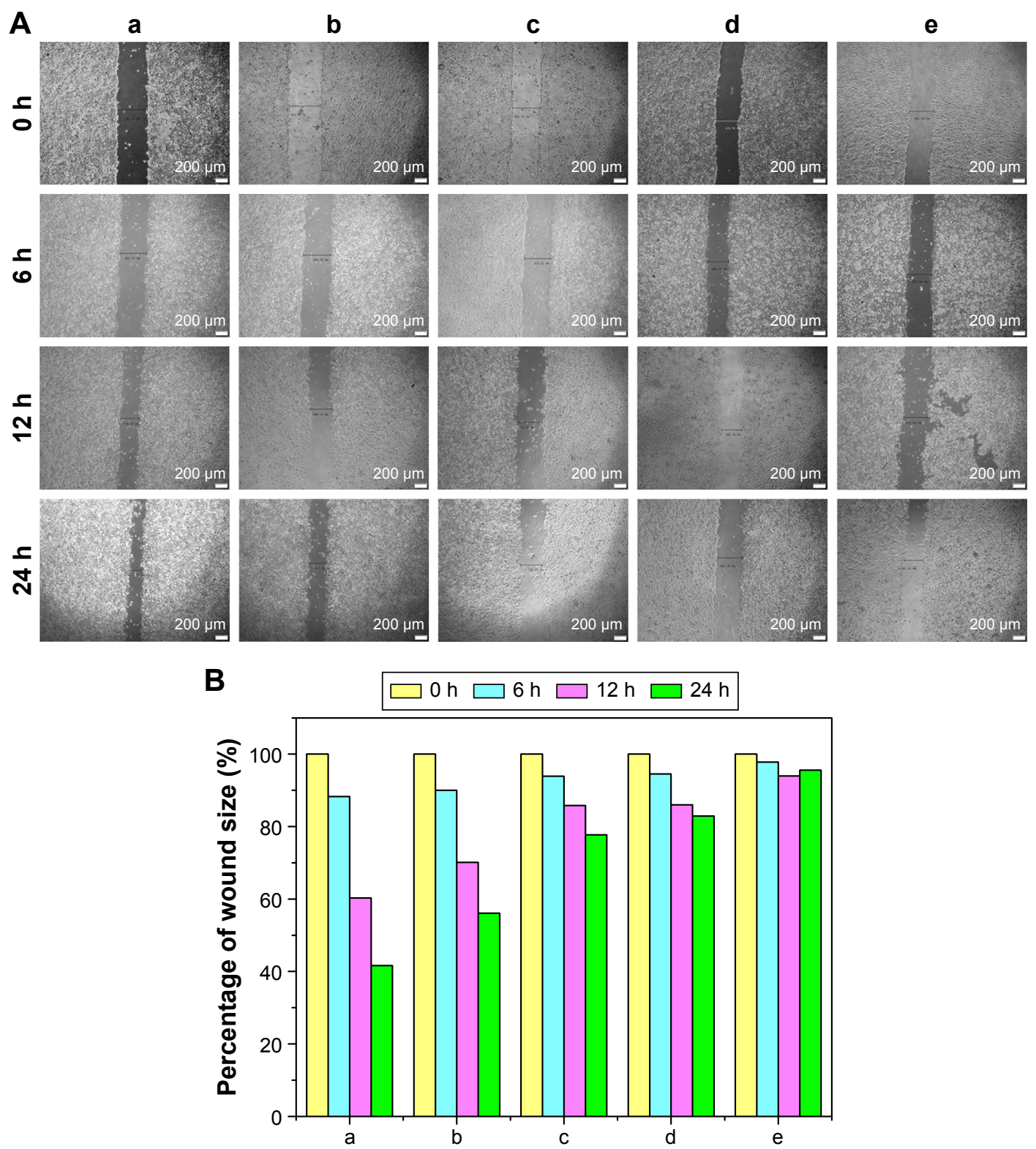

Figure 8 Wound healing (A) and quantitative assay (B) of A549 cells treated with the release supernatants from disulfiram-loaded porous poly(lactic-co-glycolic acid) microparticles for $24 \mathrm{~h}$ : (a) control; (b-e) treatment with the release supernatants at I, 3, 5, and 7 days, respectively.



Figure 9 Transwell migration assay for the antimigration effect in A549 cells treated with the release supernatants from disulfiram-loaded porous poly(lactic-co-glycolic acid) microparticles for $24 \mathrm{~h}$ : (A) control; (B-E) treatment with the release supernatants at I, 3, 5, and 7 days, respectively. 


\section{Acknowledgments}

The authors gratefully acknowledge the financial supports from Natural Science Foundation of China (Nos 81373344 and 51403074), Science and Technology Department of Jilin Province (Nos 20140101140JC, 20160520144JH, and 20160520146JH), Education Department of Jilin Province (No 2015469), the Youth Fund of Health and Family Planning Commission of Jilin Province (No 2013Q026), and Norman Bethune Program of Jilin University (Nos 2015324 and 2015423).

\section{Disclosure}

The authors report no conflicts of interest in this work.

\section{References}

1. Duan L, Shen H, Zhao G, et al. Inhibitory effect of disulfiram/copper complex on non-small cell lung cancer cells. Biochem Biophys Res Commun. 2014;446(4):1010-1016.

2. Binder D, Hegenbarth K. Emerging options for the management of nonsmall cell lung cancer. Clin Med Insights Oncol. 2013;7:221-234.

3. Sauna ZE, Shukla S, Ambudkar SV. Disulfiram, an old drug with new potential therapeutic uses for human cancers and fungal infections. Mol Biosyst. 2005;1(2):127-134.

4. Wickström M, Danielsson K, Rickardson L, et al. Pharmacological profiling of disulfiram using human tumor cell lines and human tumor cells from patients. Biochem Pharmacol. 2007;73(1):25-33.

5. Zhang H, Chen D, Ringler J, et al. Disulfiram treatment facilitates phosphoinositide 3-kinase inhibition in human breast cancer cells in vitro and in vivo. Cancer Res. 2010;70(10):3396-4004.

6. Cvek B. Targeting malignancies with disulfiram (Antabuse): multidrug resistance, angiogenesis, and proteasome. Curr Cancer Drug Targets. 2011;11(3):332-337.

7. Cheriyan VT, Wang Y, Muthu M, et al. Disulfiram suppresses growth of the malignant pleural mesothelioma cells in part by inducing apoptosis. PLoS One. 2014;9(4):e93711.

8. Duan X, Xiao J, Yin Q, et al. Multi-targeted inhibition of tumor growth and lung metastasis by redox-sensitive shell crosslinked micelles loading disulfiram. Nanotechnology. 2014;25(12):125102.

9. Nechushtan H, Hamamreh Y, Nidal S, et al. A phase IIb trial assessing the addition of disulfiram to chemotherapy for the treatment of metastatic non-small cell lung cancer. Oncologist. 2015;20(4):366-367.

10. Zha J, Chen F, Dong H, et al. Disulfiram targeting lymphoid malignant cell lines via ROS-JNK activation as well as Nrf2 and NF-kB pathway inhibition. $J$ Transl Med. 2014;12:163.

11. Song W, Tang Z, Lei T, et al. Stable loading and delivery of disulfiram with mPEG-PLGA/PCL mixed nanoparticles for tumor therapy. Nanomedicine. 2016;12(2):377-386.

12. Duan X, Xiao J, Yin Q, et al. Smart pH-sensitive and temporalcontrolled polymeric micelles for effective combination therapy of doxorubicin and disulfiram. ACS Nano. 2013;7(7):5858-5869.

13. Muralidharan P, Malapit M, Mallory E, Hayes D Jr, Mansour HM. Inhalable nanoparticulate powders for respiratory delivery. Nanomedicine. 2015; 11(5):1189-1199.
14. Hidalgo A, Cruz A, Pérez-Gil J. Barrier or carrier? Pulmonary surfactant and drug delivery. Eur J Pharm Biopharm. 2015;95(Pt A):117-127.

15. Sosnowski TR. Nanosized and nanostructured particles in pulmonary drug delivery. J Nanosci Nanotechnol. 2015;15(5):3467-3487.

16. Edwards DA, Hanes J, Caponetti G, et al. Large porous particles for pulmonary drug delivery. Science. 1997;276(5320):1868-1871.

17. Kim TK, Yoon JJ, Lee DS, Park TG. Gas foamed open porous biodegradable polymeric microspheres. Biomaterials. 2016;27(2): $152-159$.

18. Kwon MJ, Bae JH, Kim JJ, Na K, Lee ES. Long acting porous microparticle for pulmonary protein delivery. Int J Pharm. 2007; 333(1-2):5-9.

19. Yang Y, Bajaj N, Xu P, Ohn K, Tsifansky MD, Yeo Y. Development of highly porous large PLGA microparticles for pulmonary drug delivery. Biomaterials. 2009;30(10):1947-1953.

20. Ungaro F, d'Emmanuele di Villa Bianca R, Giovino C, et al. Insulinloaded PLGA/cyclodextrin large porous particles with improved aerosolization properties: in vivo deposition and hypoglycaemic activity after delivery to rat lungs. J Control Release. 2009;135(1):25-34.

21. Oh YJ, Lee J, Seo JY, et al. Preparation of budesonide-loaded porous PLGA microparticles and their therapeutic efficacy in a murine asthma model. J Control Release. 2011;150(1):56-62.

22. Kim I, Byeon HJ, Kim TH, et al. Doxorubicin-loaded highly porous large PLGA microparticles as a sustained-release inhalation system for the treatment of metastatic lung cancer. Biomaterials. 2012; 33(22):5574-5583.

23. Shi X, Li C, Gao S, et al. Combination of doxorubicin-based chemotherapy and polyethylenimine/p53 gene therapy for the treatment of lung cancer using porous PLGA microparticles. Colloids Surf B Biointerf. 2014;122:498-504.

24. Wang $\mathrm{C}, \mathrm{Wu} \mathrm{D}$, Yang $\mathrm{J}$, et al. Porous PLGA microparticles to encapsulate doxorubicin and polyethylenimine/miR-34a for inhibiting the proliferation and migration of lung cancer. $R S C A d v$. 2015; 5(99):81445-81448.

25. Han FY, Thurecht KJ, Whittaker AK, Smith MT. Bioerodable PLGAbased microparticles for producing sustained-release drug formulations and strategies for improving drug loading. Front Pharmacol. 2016;7:185.

26. Wei Y, Wang Y, Zhang H, Zhou W, Ma G. A novel strategy for the preparation of porous microspheres and its application in peptide drug loading. J Colloid Interface Sci. 2016;478:46-53.

27. Tanaka A, Fukuoka Y, Morimoto Y, et al. Cancer cell death induced by the intracellular self-assembly of an enzyme-responsive supramolecular gelator. J Am Chem Soc. 2015;137(2):770-775.

28. Lowe SW, Lin AW. Apoptosis in cancer. Carcinogenesis. 2000; 21(3):485-495.

29. Fasehee H, Zarrinrad G, Tavangar SM, Ghaffari SH, Faghihi S. The inhibitory effect of disulfiram encapsulated PLGA NPs on tumor growth: different administration routes. Mater Sci Eng C Mater Biol Appl. 2016;63:587-595.

30. Hoda M, Pajaniradje S, Shakya G, Mohankumar K, Rajagopalan R. Anti-proliferative and apoptosis-triggering potential of disulfiram and disulfiram-loaded polysorbate 80 -stabilized PLGA nanoparticles on hepatocellular carcinoma Hep3B cell line. Nanomedicine. 2016; 12(6):1641-1650.

31. Khanna KK, Jackson SP. DNA double-strand breaks: signaling, repair and the cancer connection. Nat Genet. 2001;27(3):247-254. 


\section{Supplementary materials}

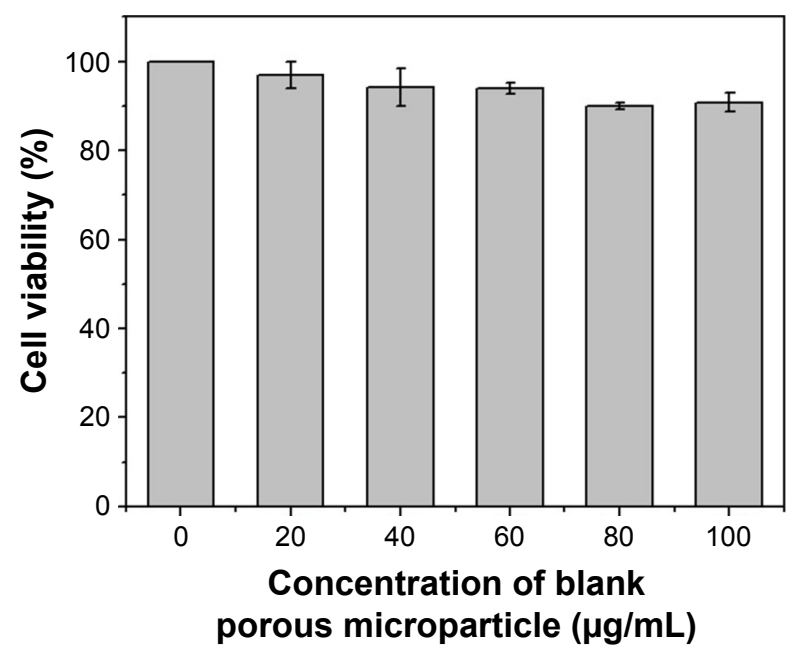

Figure SI In vitro cytotoxicity analysis of blank porous poly(lactic-co-glycolic acid) microparticle in L-02 cells. The cell viability of I00\% was set as no treatment of microparticle, and the data were expressed as mean value \pm SD of three experiments.

Abbreviation: SD, standard deviation.



Figure S2 Western blotting analysis for the expression levels of pro-caspase-3, caspase-8, and caspase-9 in A549 cells treated with the release supernatants from disulfiramloaded porous poly(lactic-co-glycolic acid) microparticles for $36 \mathrm{~h}$ : lane I: control; lanes 2-5: treatment with the release supernatants at I, 3, 5, and 7 days, respectively.

\section{Publish your work in this journal}

The International Journal of Nanomedicine is an international, peerreviewed journal focusing on the application of nanotechnology in diagnostics, therapeutics, and drug delivery systems throughou the biomedical field. This journal is indexed on PubMed Central, MedLine, CAS, SciSearch $®$, Current Contents $\AA /$ Clinical Medicine,
Journal Citation Reports/Science Edition, EMBase, Scopus and the Elsevier Bibliographic databases. The manuscript management system is completely online and includes a very quick and fair peer-review system, which is all easy to use. Visit http://www.dovepress.com/ testimonials.php to read real quotes from published authors 